УДК 519.171 .4

DOI: 10.15827/0236-235X.123.598-604

Дата подачи статьи: 26.03.18

\title{
Модель группировки радиоэлектронных систем для оченки временных показателей надежности
}

\author{
С.В. Игнатьев ${ }^{1}$, д.т.н., профессор, начальник кафедры
}

Ю.А. Плакса ${ }^{1}$, к.т.н., доиент

А.В. Красников ${ }^{1}$, к.т.н., доиент

A.В. Дрожжжин 1, адъюнкт, drozhzhin-1991@mail.ru

1 Ярославское высшее военное училище противовоздушной обороны, кафедра автоматики и вычислительных средств, г. Ярославль, 150001, Россия

Эффективное применение комплексов специального назначения, основу которых составляют радиоэлектронные системы, предполагает выбор оптимальных методов эксплуатации, организации и проведения технического обслуживания, войскового ремонта и снабжения комплексов запасными инструментами и принадлежностями с целью обеспечения высокой степени готовности этих комплексов к применению по предназначению. Для этого создана система технической эксплуатации, эффективность функционирования которой зависит от взаимного расположения радиоэлектронных систем на местности.

Основные положения работы связаны с построением модели группировки радиоэлектронных систем, которая является основой для разработки инструментальной среды, с учетом пространственного расположения элементов группировки и временных соотношений между ними.

Построение модели группировки выполняется в два этапа. Первый этапа заключается в построении модели транспортной сети местности, которая представляет собой совокупность графа и матрицы достижимости. Такое представление позволяет получить все возможные маршруты между элементами транспортной сети. На втором этапе осуществляется описание группировки радиоэлектронных систем путем выделения на графе транспортной сети вершин особого типа, в которых размещаются элементы группировки радиоэлектронных систем. Далее производится построение пространственно-временной модели группировки радиоэлектронных систем, представляющей собой совокупность графа и подматриц достижимостей, где каждому маршруту между элементами группировки ставится в соответствие временной показатель (время движения по маршруту).

Пространственно-временная модель группировки, программно реализованная на языке С\#, позволяет рассчитать временные показатели надежности с учетом влияния различных факторов и оценить степень их влияния на коэффициент готовности.

Ключевые слова: группировка радиоэлектронных систем, пространственно-временная модель, транспортная сеть местности, граф, матрица достижимости, временная система маршрутов.

Актуальность поддержания сложных радиоэлектронных систем (РЭС) на заданном уровне надежности не вызывает сомнений. Для этого создана система технической эксплуатаџии (СТЭ), которая, в свою очередь, включает системы технического обслуживания, диагностирования и материально-технического обеспечения [1]. Функционирование первых двух систем направлено на увеличение времени наработки РЭС до отказа $\left(T_{\mathrm{O}}\right)$ и уменьшение их времени восстановления $\left(T_{\mathrm{B}}\right)$ соответственно.

На сегодняшний день исследования ведутся по каждой системе, причем в разных направлениях [2-6]. В основу таких исследований положены математические модели, учитывающие влияние различных параметров этих систем на надежность РЭС. Кроме того, они проводились при определенных ограничениях, одним из которых является допущение того, что РЭС полностью укомплектованы требуемыми материально-техническими ресурсами (МТР) и последние не оказывают никакого воздействия на $T_{\mathrm{O}}$ и $T_{\mathrm{B}}$. Такой подход не позволяет в полной мере оценить эффективность функционирования СТЭ при обеспечении заданного уровня надежности группировки РЭС, что приводит к необходимости более глубокого исследования процесса восстановления РЭС.

Опыт эксплуатации показывает, что эффективность функционирования СТЭ будет зависеть от ряда факторов. Прежде всего это взаимное расположение на местности элементов группировки РЭС, что определяет пространственную топологию группировки (так называемый пространственный параметр). Кроме того, в процессе эксплуатации возникает ряд задач [7]: доставка запасных частей, инструментов и принадлежностей, горюче-смазочных материалов, прибытие специалиста для ремонта и т.д. Их решение характеризуется временными показателями, которые, в свою очередь, являются составляющими времени восстановления: $T_{\mathrm{B}}=t_{\mathrm{K}}+t_{\mathrm{p}}+t_{\text {подг. }}+t_{\text {пер. }}+t_{\text {дост. }}+t_{\text {адм. }}$, где $t_{\mathrm{K}}-$ время поиска и локализации отказавшего элемента; $t_{\mathrm{p}}$ время устранения отказа; $t_{\text {подг. }}-$ время подготовки к включению; $t_{\text {пер. }}$ - время перерывов обслуживающего персонала; $t_{\text {дост. }}-$ время доставки исправного элемента; $t_{\text {адм. }}-$ время прибытия специалиста для ремонта [1]. Как правило, решение последних двух задач осуществляется с использованием транспортной сети местности (ТСМ), на которой расположена группировка РЭС. 


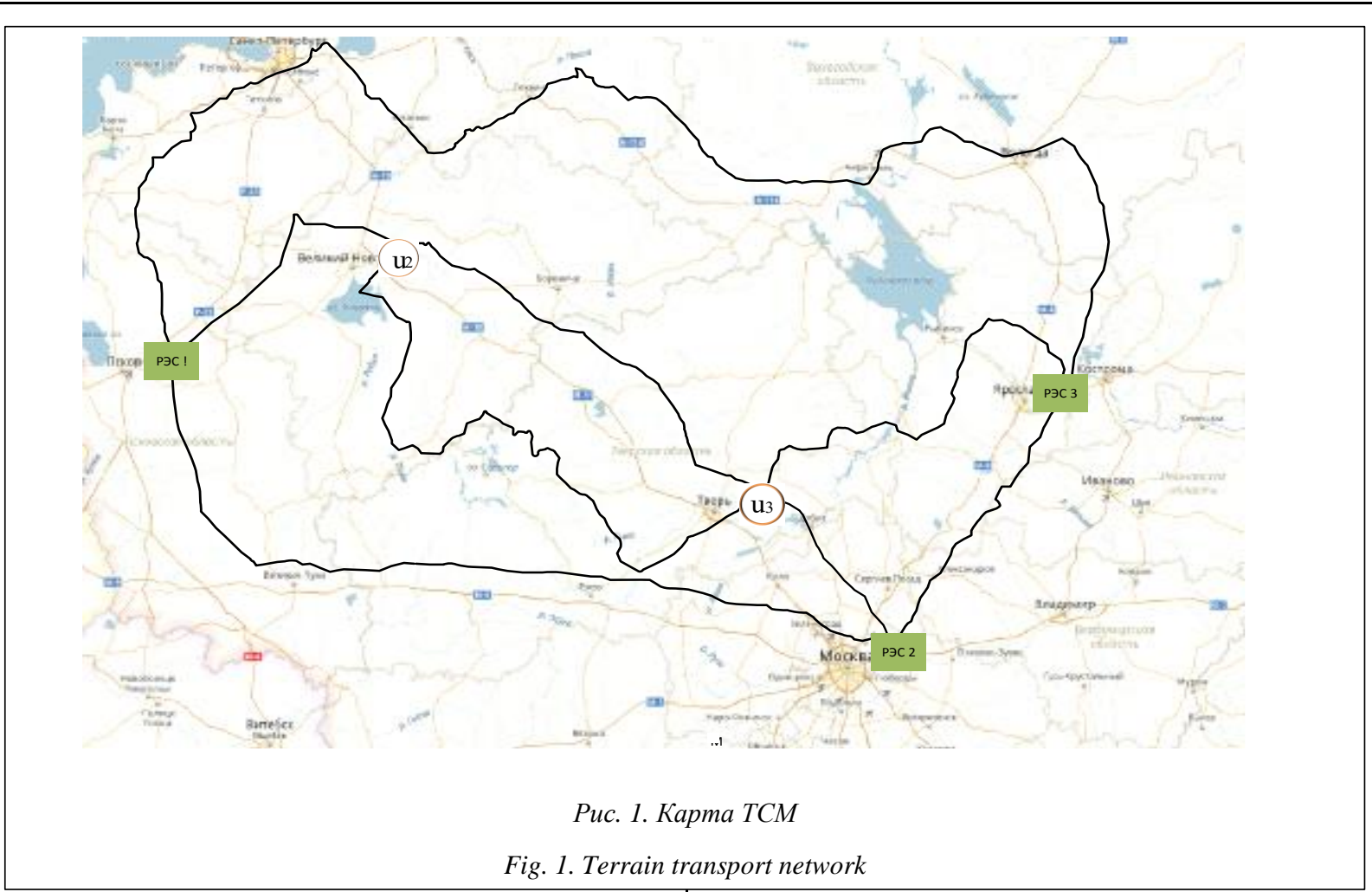

Учитывая это, время восстановления элемента группировки можно представить как $T_{\mathrm{B}}=T_{\text {Эг }}+T_{\mathrm{TC}}$, где $T_{\ni г}=t_{\mathrm{K}}+t_{\mathrm{p}}+t_{\text {подг. }}+t_{\text {пер. }}-$ время выполнения операций в элементе группировки; $T_{\mathrm{TC}}=t_{\text {дост. }}+$ $+t_{\text {адм. }}-$ время выполнения операций с использованием транспортной сети (ТС).

В этом случае расстояние $s$ между узлами ТСМ, в которых размещаются элементы группировки РЭС, и средняя скорость движения $v$ будут определять $T_{\mathrm{TC}}$ (так называемый временной параметр).

Для комплексного учета возможных ситуаций и проведения дальнейших исследований с оценкой показателей надежности, связанных с временными параметрами (коэффициент готовности, коэффициент технического использования, время восстановления), необходимо построить модель группировки РЭС, учитывающую пространственное положение ее элементов и временные соотношения между ними, которую будем называть пространственно-временной моделью группировки РЭС.

С учетом того, что группировка РЭС располагается на местности (на ТС), построение данной модели целесообразно осуществить в два этапа.

Первый этап предполагает построение модели TCM, на которой размещается группировка РЭС. На втором этапе производится описание группировки РЭС на модели ТСМ путем размещения в ее узлах элементов группировки.

\section{Первый этап (построение модели ТСМ)}

Рассмотрим абстрактный пример. Пусть имеется некая ТСМ, на которой размещается группировка РЭС (рис. 1).
Для ее формализации осуществим переход от концептуальной модели (разнотипных элементов, описанных в терминах предметной области) к ее математическому описанию (формальным однотипным). Очевидно, что наиболее просто это осуществимо с использованием теории графов [8].

Представим узловые пункты ТС (населенные пункты, элементы РЭС, пункты перегрузки груза и смены транспорта, развилки дорог и т.д.) вершинами (узлами) графа, а пути сообщения между этими пунктами (наземные, воздушные, водные) ребрами графа.

В результате получим мультиграф ТС (рис. 2): $G_{\Pi}=<U, R>$, где $U=\left\{u_{i} \mid i=1,2, \ldots, 5\right\}$ - множество вершин графа; $R=\left\{r_{k} \mid k=1,2, \ldots, 8\right\}$ - множество ребер графа [4].

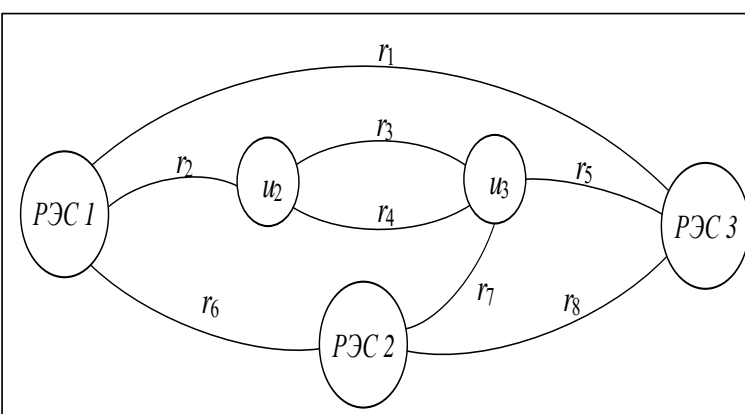

Рис. 2. Неориентированный мультиграф ТС

Fig. 2. Transport network undirected multigraph

В общем случае $G_{\Pi}=<U, R>$, где $U=\left\{u_{i} \mid i=1\right.$, $2, \ldots, I\}, I-$ количество вершин графа; $R=\left\{r_{k} \mid\right.$ $k=1,2, \ldots, K\}, K-$ количество ребер графа. 
Каждая вершина графа $G_{M}$ (рис. 3) нагружается кортежем

$$
e_{i}=\left[t_{i}, c_{i}\right],
$$

где $t_{i}-$ время выполнения определенных действий (время задержки) в $i$-м узле ТС (например: дозаправка транспортного средства (ТСp), оформление необходимых документов, перегрузка материальных ресурсов из одного ТСр в другое и т.д.), которые необходимо выполнить, чтобы продолжить дальнейшее движение; $c_{i}-$ стоимость выполнения этих действий в $i$-м узле ТС.

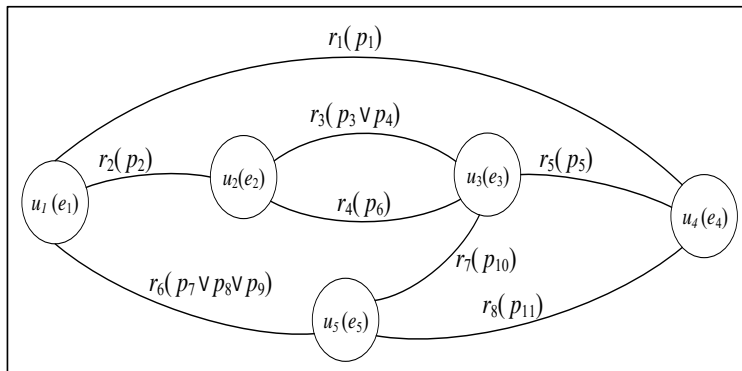

Puc. 3. Граф МTC

Fig. 3. Graph of transport route network

Ребра графа $G_{M}$ нагружены кортежем

$$
p=\left[r_{k}, s_{k}, \theta_{n}, v_{k}\left(\theta_{n}\right), g_{k}, c_{k}\left(\theta_{n}\right)\right],
$$

где $r_{k}$ - обозначение участка пути ТС (ребра графа); $s_{k}$ - протяженность участка пути ТС; $\theta_{n}-$ тип ТСp, движущегося по участку пути ТС; $v_{k}\left(\theta_{n}\right)-$ скорость $\mathrm{TCp}$, движущегося по участку пути ТС; $g_{k}-$ коэффициент изменения скорости ТСp; $c_{k}\left(\theta_{n}\right)-$ стоимость перевозки конкретным типом ТСр за единицу пути.

Исходя из вышеизложенного, в общем виде граф маршрутов ТС [8,9] опишется выражением

$G_{\mathrm{m}}=\langle(U, E) ;(R, P)\rangle$,

$U=\left\{u_{1}, u_{2}, \ldots, u_{i}, \ldots, u_{I}\right\}$,

$E=\left\{e_{1}, e_{2}, \ldots, e_{i}, \ldots e_{I}\right\}$,

$R=\left\{r_{1}, r_{2}, \ldots, r_{k}, \ldots, r_{K}\right\}$,

$P=\left\{p_{1}, p_{2}, \ldots, p_{l}, \ldots, p_{L}\right\}$

$e_{i}=\left[t_{i}, c_{i}\right], p_{l}=\left[r_{k}, s_{k}, \theta_{n}, v_{k}\left(\theta_{n}\right), g_{k}, c_{k}\left(\theta_{n}\right)\right]$,

$\theta_{n} \in \Theta, n=1,2, \ldots, N$.

Веса вершин и ребер графа (рис. 3) могут выступать в качестве их идентификаторов. Поэтому $G_{\mathrm{M}}$ опишем матрицей смежности взвешенного графа [9-11] (весовой матрицей смежности) $S\left(G_{\mathrm{M}}\right)=\left[s_{i j}\right]_{I \times I}$ вида

$$
s_{i j}=\left\{\begin{array}{l}
p_{l}, \text { если ребро } r_{k} \in R, \\
0, \text { если ребро } r_{k} \notin R .
\end{array}\right.
$$

В результате весовая матрица смежности $S\left(G_{\mathrm{M}}\right)$ для рассматриваемого графа будет иметь следующий вид:

$S\left(G_{\mathrm{u}}\right)=$\begin{tabular}{|c|c|c|c|c||}
\hline & $p_{2}$ & & $p_{1}$ & $\left(p_{7} \vee p_{8} \vee p_{9}\right)$ \\
\hline$p_{2}$ & & $\left(p_{3} \vee p_{4} \vee p_{6}\right)$ & & \\
\hline & $\left(p_{3} \vee p_{4} \vee p_{6}\right)$ & & $p_{5}$ & $p_{10}$ \\
\hline$p_{1}$ & & $p_{5}$ & & $p_{11}$ \\
\hline$\left(p_{7} \vee p_{8} \vee p_{9}\right)$ & & $p_{10}$ & $p_{11}$ & \\
\hline
\end{tabular}

Для получения возможных маршрутов между $i$-й и $j$-й вершинами графа $G_{M}-m_{i j}$, где $i=\overline{1,5}$, $j=\overline{1,5}$ и $i \neq j$, находится матрица достижимости:

$$
\begin{aligned}
& D(G)=\sum_{n=1}^{d(G)}[S(G)]^{n}= \\
& =S(G)+S(G)^{2}+\ldots S(G)^{n}+\ldots+S(G)^{d(G)},
\end{aligned}
$$

где $d(G)$ - диаметр графа $G_{\mathrm{M}}$.

При этом операцию умножения при возведении матрицы смежности в степень необходимо рассматривать как конкатенацию, а операцию сложения - как дизъюнкцию и учитывать только простые маршруты, в которых отсутствуют повторения вершин графа между начальным $e_{i}$ и конечным $e_{j}$ узлами маршрута: $e_{i} \neq \ldots \neq e_{q} \neq \ldots e_{j}$, то есть каждая вершина в одном маршруте должна встречаться один раз.

В общем виде

$$
\begin{aligned}
& D\left(G_{M}\right)=\bigvee_{n=1}^{d(G)}\left[S\left(G_{M}\right)\right]^{n}= \\
& =S\left(G_{M}\right) \vee S\left(G_{M}\right)^{2} \vee \ldots \vee S\left(G_{M}\right)^{n} \vee \ldots \vee S\left(G_{M}\right)^{d(G)} .
\end{aligned}
$$

Так, например, в соответствии с выражением (7) маршруты между узлами $e_{1}$ и $e_{2}$ опишутся следующим выражением:

$$
\begin{aligned}
& m_{1,2}=e_{1} p_{2} e_{2} \vee e_{1} p_{1} e_{4} p_{5} e_{3}\left(p_{3} \vee p_{4} \vee p_{6}\right) e_{2} \vee \\
& \vee e_{1}\left(p_{7} \vee p_{8} \vee p_{9}\right) e_{5} p_{10} e_{3}\left(p_{3} \vee p_{4} \vee p_{6}\right) e_{2} \vee \\
& \vee e_{1}\left(p_{7} \vee p_{8} \vee p_{9}\right) e_{5} p_{11} e_{4} p_{5} e_{3}\left(p_{3} \vee p_{4} \vee p_{6}\right) e_{2} \vee \\
& \vee e_{1} p_{1} e_{4} p_{11} e_{5} p_{10} e_{3}\left(p_{3} \vee p_{4} \vee p_{6}\right) e_{2} .
\end{aligned}
$$

Тогда с учетом примера (8) представим матрицу достижимости:

$$
D\left(G_{\mathrm{M}}\right)=\left\|\begin{array}{||l|l|l|l|l}
\hline & m_{1,2} & m_{1,3} & m_{1,4} & m_{1,5} \\
\hline m_{2,1} & & m_{2,3} & m_{2,4} & m_{2,5} \\
\hline m_{3,1} & m_{3,2} & & m_{3,4} & m_{3,5} \\
\hline m_{4,1} & m_{4,2} & m_{4,3} & & m_{4,5} \\
\hline m_{5,1} & m_{5,2} & m_{5,3} & m_{5,4} & \\
\hline
\end{array}\right\| .
$$

Содержимое ячеек матрицы (9) полностью определяет систему маршрутов ТС $[4,9]$.

В общем виде модель ТС запишется как $G_{\mathrm{м}}=\langle(U, E) ;(R, P)\rangle, U=\left\{u_{1}, u_{2}, \ldots, u_{i}, \ldots, u_{I}\right\}$, $E=\left\{e_{1}, e_{2}, \ldots, e_{i}, \ldots e_{I}\right\}, R=\left\{r_{1}, r_{2}, \ldots, r_{k}, \ldots, r_{K}\right\}$, $P=\left\{p_{1}, p_{2}, \ldots, p_{l}, \ldots, p_{L}\right\}$,

$e_{i}=\left[t_{i}, c_{i}\right], p_{l}=\left[r_{k}, s_{k}, \theta_{n}, v_{k}\left(\theta_{n}\right), g_{k}, c_{k}\left(\theta_{n}\right)\right]$, $\theta_{n} \in \Theta$. 


$$
D\left(G_{\mathrm{M}}\right)=\left\{\begin{array}{l}
m_{i, j}=\bigvee_{h}\left(e_{i} p_{x_{h}} e_{a_{h}} \ldots p_{y_{h}} \ldots e_{b_{h}} p_{z_{h}} e_{j}\right)_{h} ; \\
h=1,2, \ldots H_{i j} ; \\
\forall h: e_{i} \neq e_{a_{h}} \neq \ldots \neq e_{b_{h}} \neq e_{j} ; \\
e_{i}, e_{a_{h}}, e_{b_{h}}, e_{j} \in E ; \\
p_{x_{h}}, p_{y_{h}} \in P ; i=1,2, \ldots, I ; j=1,2, \ldots, I ; \\
\vdots \\
m_{i, j}=\bigvee_{h}\left(e_{i} p_{x_{h}} e_{a_{h}} \ldots \vee \ldots e_{b_{h}} p_{y_{h}} e_{j}\right)_{h} ; \\
h=1,2, \ldots ; \\
\forall h: e_{i} \neq e_{a_{h}} \neq \ldots \neq e_{b_{h}} \neq e_{j} ; e_{i}, e_{a_{h}}, e_{b_{h}}, e_{j} \in E ; \\
p_{x_{h}}, p_{y_{h}} \in P ; j=1,2, \ldots, I ; i=1,2, \ldots, I ; i \neq j .
\end{array}\right.
$$

В соответствии с выражением (10) количество маршрутов между начальным $e_{i}$ и конечным $e_{j}$ узлами ТС $H_{i j}$ будет равно количеству слагаемых по операции дизъюнкции.

\section{Второй этап (описание группировки РЭС на ТСМ)}

Для описания группировки РЭС выделим из рассматриваемого графа ТС вершины особого типа, в которых размещаются элементы группировки РЭС, и объединим их в множество $E^{\prime}: E^{\prime} \subseteq E$. Пусть для рассматриваемого графа ТС (рис. 2) это вершины $e_{1}, e_{4}, e_{5}: E^{\prime}=e_{1}, e_{4}, e_{5}$. В результате получим граф группировки РЭС (рис. 4).

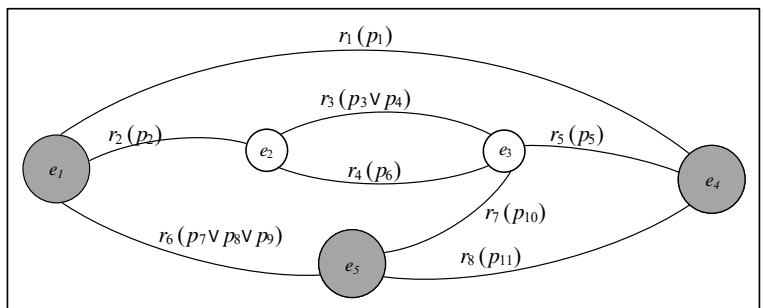

Рис. 4. Граф группировки РЭС

Fig. 4. Graph of radio-electronic system nest

Особенностью данных вершин является то, что попарное сочетание вершин множества образуют начало и конец маршрутов ТС. Другие вершины графа (рис. 4) не могут выступать в качестве начала и окончания маршрутов.

Матрица достижимости $D^{\prime}\left(G_{\mathrm{M}}\right)$ для вершин графа из множества $E^{\prime}$ получается из $D\left(G_{\mathrm{M}}\right)$ путем вычисления соответствующих строк и столбцов:

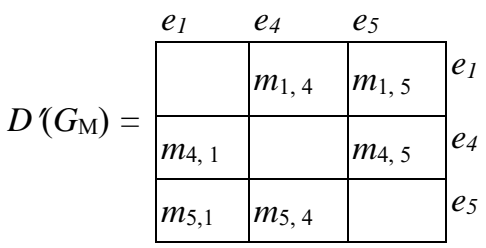

Следует отметить, что полученная подматрица является симметричной. Учтем сделанные ранее замечания (вес вершины (ребра) при движении в прямом и обратном направлениях одинаковый).
Для уменьшения количества вычислений будем использовать только те ячейки преобразованной матрицы достижимости, которые находятся выше (ниже) главной диагонали. Тогда система маршрутов между элементами группировки для рассматриваемого примера примет вид

$$
\begin{aligned}
& \mid \begin{array}{l}
m_{1}=e_{1} p_{1} e_{4} \\
m_{2}=e_{1} p_{7} e_{5} p_{11} e_{4} ;
\end{array} \\
& m_{3}=e_{1} p_{8} e_{5} p_{11} e_{4} ; \\
& m_{4}=e_{1} p_{9} e_{5} p_{11} e_{4} ; \\
& m_{5}=e_{1} p_{2} e_{2} p_{3} e_{3} p_{5} e_{4} ; \\
& m_{6}=e_{1} p_{2} e_{2} p_{4} e_{3} p_{5} e_{4} ; \\
& m_{1,4}=\left\{m_{7}=e_{1} p_{2} e_{2} p_{6} e_{3} p_{5} e_{4} ;\right. \\
& m_{8}=e_{1} p_{7} e_{5} p_{10} e_{3} p_{5} e_{4} ; \\
& m_{9}=e_{1} p_{8} e_{5} p_{10} e_{3} p_{5} e_{4} ; \\
& m_{10}=e_{1} p_{9} e_{5} p_{10} e_{3} p_{5} e_{4} ; \\
& m_{11}=e_{1} p_{2} e_{2} p_{3} e_{3} p_{10} e_{5} p_{11} e_{4} \text {; } \\
& m_{12}=e_{1} p_{2} e_{2} p_{4} e_{3} p_{10} e_{5} p_{11} e_{4} \text {; } \\
& m_{13}=e_{1} p_{2} e_{2} p_{6} e_{3} p_{10} e_{5} p_{11} e_{4} \text {. } \\
& \left\{\begin{array}{l}
m_{1}=e_{1} p_{7} e_{5} ; \\
m_{2}=e_{1} p_{8} e_{5} ;
\end{array}\right. \\
& m_{3}=e_{1} p_{9} e_{5} ; \\
& m_{4}=e_{1} p_{1} e_{4} p_{11} e_{5} ; \\
& m_{1,5}=\left\{\begin{array}{l}
m_{5}=e_{1} p_{2} e_{2} p_{3} e_{3} p_{10} e_{5} ; \\
m_{6}=e_{1} p_{2} e_{2} p_{4} e_{3} p_{10} e_{5} ;
\end{array} ;\right. \\
& m_{7}=e_{1} p_{2} e_{2} p_{6} e_{3} p_{10} e_{5} ; \\
& m_{8}=e_{1} p_{1} e_{4} p_{5} e_{3} p_{10} e_{5} ; \\
& m_{9}=e_{1} p_{2} e_{2} p_{3} e_{3} p_{5} e_{4} p_{11} e_{5} ; \\
& m_{10}=e_{1} p_{2} e_{2} p_{4} e_{3} p_{5} e_{4} p_{11} e_{5} ; \\
& m_{11}=e_{1} p_{2} e_{2} p_{6} e_{3} p_{5} e_{4} p_{11} e_{5} \\
& m_{1}=e_{4} p_{11} e_{5} \text {; } \\
& m_{2}=e_{4} p_{1} e_{1} p_{7} e_{5} ; \\
& m_{3}=e_{4} p_{1} e_{1} p_{8} e_{5} ; \\
& m_{4}=e_{4} p_{1} e_{1} p_{9} e_{5} ; \\
& m_{5}=e_{4} p_{5} e_{3} p_{10} e_{5} ; \\
& m_{6}=e_{4} p_{5} e_{3} p_{3} e_{2} p_{2} e_{1} p_{7} e_{5} \\
& m_{7}=e_{4} p_{5} e_{3} p_{3} e_{2} p_{2} e_{1} p_{8} e_{5} \\
& m_{4,5}=\left\{\begin{array}{l}
m_{8}=e_{4} p_{5} e_{3} p_{3} e_{2} p_{2} e_{1} p_{9} e_{5} ; \\
m_{9}=e_{4} p_{5} e_{3} p_{4} e_{2} p_{2} e_{1} p_{7} e_{5} ;
\end{array}\right. \\
& m_{10}=e_{4} p_{5} e_{3} p_{4} e_{2} p_{2} e_{1} p_{8} e_{5} ; \\
& m_{11}=e_{4} p_{5} e_{3} p_{4} e_{2} p_{2} e_{1} p_{9} e_{5} ; \\
& m_{12}=e_{4} p_{5} e_{3} p_{6} e_{2} p_{2} e_{1} p_{7} e_{5} ; \\
& m_{13}=e_{4} p_{5} e_{3} p_{6} e_{2} p_{2} e_{1} p_{8} e_{5} ; \\
& m_{14}=e_{4} p_{5} e_{3} p_{6} e_{2} p_{2} e_{1} p_{9} e_{5} ; \\
& m_{15}=e_{4} p_{1} e_{1} p_{2} e_{2} p_{3} e_{3} p_{10} e_{5} ; \\
& m_{16}=e_{4} p_{1} e_{1} p_{2} e_{2} p_{4} e_{3} p_{10} e_{5} ; \\
& m_{17}=e_{4} p_{1} e_{1} p_{2} e_{2} p_{6} e_{3} p_{10} e_{5}
\end{aligned}
$$

Для дальнейшего решения задачи преобразованную матрицу достижимости представим в виде набора подматриц достижимостей, в ячейках которых между вершинами $e_{i}$ и $e_{j}$ записывается только 
один $h$-й маршрут $m_{i j}^{h}$, где $h-$ номер маршрута в системе $m_{i j}$. Например, для рассматриваемого примера одна из набора подматриц достижимости будет иметь вид

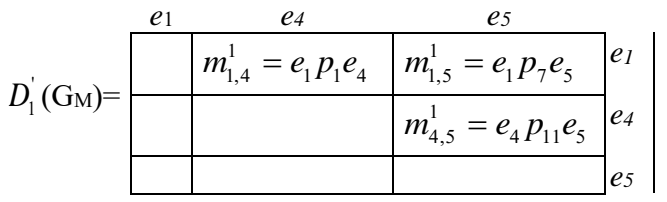

Из выражения (12) видно, что между вершинами $e_{1}$ и $e_{4}$ существуют 13 маршрутов, то есть $H_{1,4}=13$. Аналогично $H_{1,5}=11, H_{4,5}=17$. Тогда общее количество маршрутов между элементами группировки может быть вычислено как $H=H_{1,5}+$ $+H_{4,5}=41$.

В общем виде выражение для определения всех маршрутов между элементами группировки представим в виде

$$
H=\sum_{H_{i j}} \sum_{h} m_{i j}^{h} .
$$

Учитывая, что в одной ячейке подматрицы достижимости записывается один маршрут и выбор маршрута является (может быть) произвольным (осуществляется в произвольном порядке), количество подматриц достижимости $D^{\prime}\left(G_{\mathrm{M}}\right)$ для рассматриваемого примера определяется как число сочетаний из общего количества маршрутов по количеству элементов группировки: $Z=\frac{41 !}{(41-3) ! 3 !}=10660$ и в общем виде представлено как

$$
Z=C_{e^{\prime}}^{H},
$$

где $e^{\prime}-$ количество вершин особого типа (элементов группировки).

Для рассматриваемого примера набор подматриц достижимости представим в матричной форме:

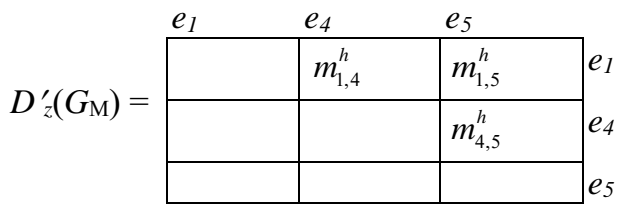

или в виде системы

$$
D_{z}^{\prime}\left(G_{\mathrm{M}}\right)=\left\{\begin{array}{l}
m_{i j}=\left(e_{i} p_{x_{h}} e_{a_{h}} \ldots p_{y_{h}} \ldots e_{b_{h}} p_{z_{h}} e_{j}\right)_{h} ; \\
h=1,2, \ldots H_{i j} ; \forall h: e_{i} \neq e_{a_{h}} \neq \ldots \neq e_{b_{h}} \neq e_{j} ; \\
e_{i}, e_{j} \in E^{\prime} \mid E^{\prime} \subseteq E ; \\
p_{x_{h}}, p_{y_{h}} \in P ; i=1,2, \ldots, I ; j=1,2, \ldots, I ; \\
\vdots \\
m_{i j}=\left(e_{i} p_{x_{h}} e_{a_{h}} \ldots \vee \ldots e_{b_{h}} p_{y_{h}} e_{j}\right)_{h} ; \\
h=1,2, \ldots H_{i j} ; \forall h: e_{i} \neq e_{a_{h}} \neq \ldots \neq e_{b_{h}} \neq e_{j} ; \\
e_{i}, e_{j} \in E^{\prime} \mid E^{\prime} \subseteq E ; \\
p_{x_{h}}, p_{y_{h}} \in P ; i=1,2, \ldots, I ; j=1,2, \ldots, I ; \\
z \in Z, Z=C_{e^{\prime}}^{H}, \\
H=\sum_{H_{i j}} \sum_{h} m_{i j}, e \in E^{\prime} \mid E^{\prime} \subseteq E .
\end{array}\right.
$$

Для каждого $h$-го маршрута системы рассчитывается $\mathrm{T}_{\mathrm{Tc}}$. Необходимые данные для этого содержатся в весах графа $e$ и $p$. Время движения на $n$-м типе ТС $\theta_{n}$ на $k$-м участке маршрута будет определяться как $t_{\text {движ. }}^{k}=\frac{S_{k}}{v_{k}\left(\theta_{n}\right) g_{k}}$, а время задержки в $i$-м узле маршрута - временем $t_{i}$.

Тогда общее время выполнения операций на всем маршруте ТС найдем как сумму соответствующих величин исходя из количества участков на этом маршруте и количества узлов, входящих в этот маршрут.

Например, для маршрута $m_{1}$ между вершинами $e_{1}$ и $e_{4}$ (см. (13)) получим следующее значение времени $\mathrm{T}_{\mathrm{TC}}$ :

$$
T_{T C_{1,4}}^{1}=t_{1}+\frac{s_{1}}{v_{1}\left(\theta_{1}\right) g_{1}}+t_{4}=t_{1}+t_{\text {дбиж }}^{1}+t_{4} .
$$

Учитывая описание маршрута выражением (20), систему маршрутов на примере выражения (12) можно представить временной системой марирутов:

$$
\begin{aligned}
& T_{m c 1}=t_{1}+t_{\text {движ. }}^{1}+t_{4} \text {; } \\
& T_{m c 2}=t_{1}+t_{\text {движ. }}^{7}+t_{5}+t_{\text {движ. }}^{11}+t_{4} \text {; } \\
& T_{\text {mс3 }}=t_{1}+t_{\text {движ. }}^{8}+t_{5}+t_{\text {движ. }}^{11}+t_{4} \text {; } \\
& T_{\text {mс4 }}=t_{1}+t_{\text {движ. }}^{9}+t_{5}+t_{\text {движ. }}^{11}+t_{4} \text {; } \\
& T_{m c 5}=t_{1}+t_{\text {движ. }}^{2}+t_{2}+t_{\text {движ. }}^{3}+t_{3}+t_{\text {движ. }}^{5}+t_{4} \text {; } \\
& T_{m c 6}=t_{1}+t_{\text {движ. }}^{2}+t_{2}+t_{\text {движ. }}^{4}+t_{3}+t_{\text {движ. }}^{5}+t_{4} \text {; } \\
& T_{T C_{1,4}}=\left\{T_{m c 7}=t_{1}+t_{\text {движ. }}^{2}+t_{2}+t_{\text {движ. }}^{6}+t_{3}+t_{\text {движ. }}^{5}+t_{4} ;\right. \\
& T_{m c 8}=t_{1}+t_{\text {движ. }}^{7}+t_{5}+t_{\text {движ. }}^{10}+t_{3}+t_{\text {движ. }}^{5}+t_{4} \text {; } \\
& T_{\text {mс9 }}=t_{1}+t_{\text {движ. }}^{8}+t_{5}+t_{\text {двик. }}^{10}+t_{3}+t_{\text {движ. }}^{5}+t_{4} \text {; } \\
& T_{m с 10}=t_{1}+t_{\text {движ. }}^{9}+t_{5}+t_{\text {движ. }}^{10}+t_{3}+t_{\text {движ. }}^{5}+t_{4} \text {; } \\
& T_{m с 11}=t_{1}+t_{\text {движ. }}^{2}+t_{2}+t_{\text {движ. }}^{3}+t_{3}+t_{\text {двнж. }}^{10}+t_{5}+t_{\text {движ. }}^{11}+t_{4} \text {; } \\
& T_{\text {mс12 }}=t_{1}+t_{\text {двик. }}^{2}+t_{2}+t_{\text {движ. }}^{4}+t_{3}+t_{\text {движ. }}^{10}+t_{5}+t_{\text {двик. }}^{11}+t_{4} \text {; } \\
& T_{m c 13}=t_{1}+t_{\text {движ. }}^{2}+t_{2}+t_{\text {движ. }}^{6}+t_{3}+t_{\text {движ. }}^{10}+t_{5}+t_{\text {движ. }}^{11}+t_{4} .
\end{aligned}
$$

В общем виде $T_{\mathrm{TC}}$ между вершинами $e_{i}$ и $e_{j}$ на $h$-м маршруте запишется как

$$
T_{T C_{i, j}}^{h}=\sum_{i} t_{i}+\sum_{k} \frac{S_{k}}{v_{k}\left(\theta_{n}\right) g_{k}}=\sum_{i} t_{i}+\sum_{k} t_{\text {движ. }}^{k} .
$$

В итоге получим пространственно-временную модель группировки РЭС $H$, представляющую собой совокупность графа (3) и подматриц достижимостей (19), где каждому $h$-му маршруту между вершинами $e_{i}$ и $e_{j}$ ставится в соответствие время $T_{\mathrm{TC}}$ на этом маршруте.

В общем виде пространственно-временная модель группировки РЭС запишется как $H=G_{M}$, $D_{z}^{\prime \prime}\left(G_{M}\right)$, где

$$
\begin{aligned}
& G_{\mathrm{M}}=\langle(U, E) ;(R, P)\rangle, U=\left\{u_{1}, u_{2}, \ldots, u_{i}, \ldots, u_{I}\right\}, \\
& E=\left\{e_{1}, e_{2}, \ldots, e_{i}, \ldots e_{I}\right\}, R=\left\{r_{1}, r_{2}, \ldots, r_{k}, \ldots, r_{K}\right\}, \\
& P=\left\{p_{1}, p_{2}, \ldots, p_{l}, \ldots, p_{L}\right\}, \\
& e_{i}=\left[t_{i}, c_{i}\right], p_{l}=,\left[r_{k}, s_{k}, \theta_{n}, v_{k}\left(\theta_{n}\right), g_{k}, c_{k}\left(\theta_{n}\right)\right], \\
& \theta_{n} \in \Theta ;
\end{aligned}
$$




$$
D_{z}^{\prime \prime}(G \mathrm{M})=\left\{\begin{array}{l}
m_{i j}=\left(e_{i} p_{x_{h}} e_{a_{h}} \ldots p_{y_{h}} \ldots e_{b_{h}} p_{z_{h}} e_{j}\right)_{h}, \\
T_{T C_{i, j}}^{h}=\sum_{i} t_{i}+\sum_{k} t_{\text {движ. }}^{k} ; \\
h=1,2, \ldots H_{i j} ; \forall h: e_{i} \neq e_{a_{h}} \neq \ldots \neq e_{b_{h}} \neq e_{j} ; \\
e_{i}, e_{j} \in E^{\prime} \mid E^{\prime} \subseteq E ; \\
p_{x_{h}}, p_{y_{h}} \in P ; i=1,2, \ldots, I ; j=1,2, \ldots, I ; \\
\vdots \\
m_{i j}=\left(e_{i} p_{x_{h}} e_{a_{h}} \ldots \vee \ldots e_{b_{h}} p_{y_{h}} e_{j}\right)_{h}, \\
T_{T C_{i, j}}^{h}=\sum_{i} t_{i}+\sum_{k} t_{\text {движ. }}^{k} ; \\
h=1,2, \ldots H_{i j} ; \forall h: e_{i} \neq e_{a_{h}} \neq \ldots \neq e_{b_{h}} \neq e_{j} ; \\
e_{i}, e_{j} \in E^{\prime} \mid E^{\prime} \subseteq E ; \\
p_{x_{h}}, p_{y_{h}} \in P ; i=1,2, \ldots, I ; j=1,2, \ldots, I ; \\
z \in Z, Z=C_{e^{\prime}}^{H}, H=\sum_{H_{i j}} \sum_{h} m_{i j}, e \in E^{\prime} \mid E^{\prime} \subseteq E .
\end{array}\right.
$$

Модель программно реализована на языке программирования С\# (получены свид. о рег. прогр. для ЭВМ № 2015661720 и № 2017616840). Интерфейс представлен на рисунках (см. рис. 5 и http:// www.swsys.ru/uploaded/image/2018-3/2018-3-dop/ 13.jpg, http://www.swsys.ru/uploaded/image/20183/2018-3-dop/14.jpg, http://www.swsys.ru/uploaded/ image/2018-3/2018-3-dop/15.jpg).

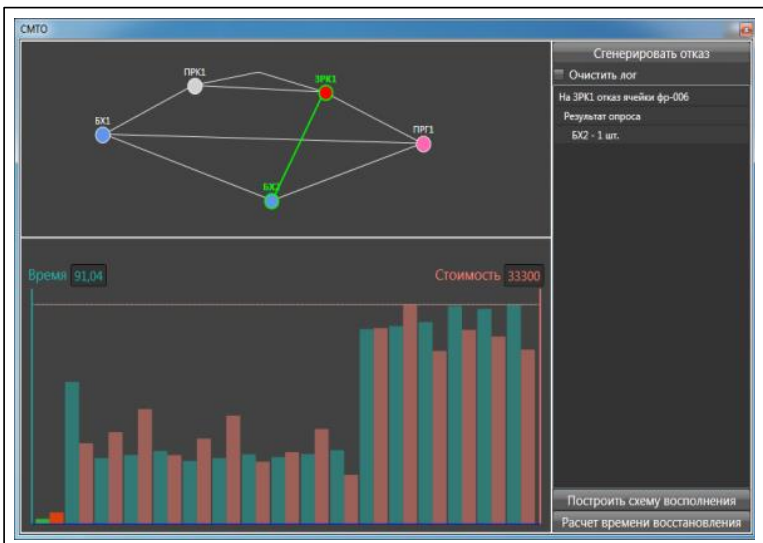

Рис. 5. Пример работы программы по расчету времени и стоимости движения

Fig. 5. The example of program operation in calculating movement time and cost
Использование данного программного продукта позволяет рассчитывать временные показатели надежности группировки РЭС и проводить исследования с учетом влияния на них следующих факторов: расположение элементов группировки на местности, расстояние между элементами группировки, тип используемого транспорта, скорость движения, условия, в которых осуществляется движение, время выполнения работ.

\section{Литература}

1. Анисимов О.В., Беляков Р.А., Игнатьев С.В., Каменский И.Е., Мещеряков В.Д., Приступюк А.И., Тихонов В.Б., Черваков В.О. Основы эксплуатации радиотехнических систем. Ярославль: Изд-во Филиала ВКА им. А.Ф. Можайского, 2015. $200 \mathrm{c}$.

2. Давыдов П.С. Техническая диагностика радиоэлектронных систем. М.: Радио и связь, 1988. 256 с.

3. Дорохов А.Н., Керножицкий В.А., Миронов А.Н., Шестопалова О.Л. Обеспечение надежности сложных технических систем. СПб: Лань, 2016. 352 с.

4. Анисимов О.В., Осипов А.А., Харитонов А.В., Беляков Р.А. Техническое обслуживание зенитного ракетного вооружения в системе ВКО // Вестн. ВУНЦ ВВС. 2012. Вып. 15. Ч. 1. C. $34-38$

5. Анисимов О.В., Попов Т.А. Метод информационной поддержки процесса диагностирования сложных технических комплексов на основе паттернов элементов электрических схем радиоэлектронной аппаратуры // Теоретические и прикладные проблемы развития и совершенствования автоматизированных систем управления военного назначения: сб. тез. II Всерос. науч.-технич. конф. СПб: 2015. С. 16-17.

6. Анисимов О.В., Курчидис В.А., Приветень А.С. Дескриптивная модель радиоэлектронной аппаратуры на основе онтологий для автоматизации информационной поддержки обслуживающего персонала при диагностировании сложных технических комплексов // Наукоемкие технологии в космических исследованиях Земли. 2016. Т. 8. № 3. С. 72-77.

7. Медведев В.М., Мищенко В.И., Солоха Н.Г. Развитие концепции эксплуатации изделий // Вестн. ОГУ. 2008. № 85. C. $149-157$.

8. Черкесов Г.Н. Оценка надежности систем с учетом ЗИП. СПб: БХВ-Петербург, 2012. 480 с.

9. Игнатьев С.В., Тихонов В.Б., Красников А.В., Осипов А.А. Пространственно-временная модель транспортной сети системы материально-технического обеспечения эксплуатации группировки радиоэлектронных средств // Программные продукты и системы. 2017. № 3. С. 510-517.

10. Оре О. Теория графов. М.: Наука, 1980. 336 с.

11. Горбатов В.А., Горбатов А.В., Горбатова М.В. Дискретная математика. М.: Астрель, 2003. 447 с.

12. Кристофидес Н. Теория графов. Алгоритмический подход. М.: Мир, 1978. 432 с.
Software \& Systems

DOI: $10.15827 / 0236-235 X .123 .598-604$
Received 26.03.18

2018, vol. 31 , no. 3 , pp. 598-604

\section{A nested model of radio-electronic systems for estimation of temporary reliability}

S.V. Ignatev ${ }^{1}$, Dr.Sc. (Engineering), Professor, Head of Chair

Yu.A. Plaksa ${ }^{1}$, Ph.D. (Engineering), Associate Professor

A.V. Krasnikov ${ }^{1}$, Ph.D. (Engineering), Associate Professor

A.V.Drozhin ${ }^{1}$, adjunct,drozhzhin-1991@mail.ru

${ }^{1}$ Yaroslavl Higher Military Air Defense College, Yaroslavl, 150001, Russian Federation

Abstract. The effective intended application of special-purpose complexes based on radio-electronic systems involves a proper choice of optimal exploitation methods, as well as organization of maintenance, first line repair and supply of the sys- 
tems with replacement tools and supplies to provide high readiness of the systems for using as intended. For this purpose, there is a system of maintenance with the operating effectiveness depending on the relative position of the radio-electronic systems in a terrain.

The essence of the article implies the construction of a nested model of radio-electronic systems designed to develop environment tools allowing to estimate temporary reliability characteristics of the nested radio-electronic systems as well as to undertake the study taking into account a relative position of nest elements and temporary correlation between them.

The construction of the nest model has two stages. The first one is the construction of a terrain transport network representing a combination of a graph and a reachability matrix. The approach allows obtaining all possible routes between transport network elements. The second stage includes a description of radio-electronic nest systems by highlighting special type apexes in the transportation network, which include nest radio-electronic systems elements. Then there goes the construction of a spatial and temporary nested model of radio-electronic systems that represens the combination of a graph and reachability submatrixes with each route being relevant of temporary features (the route movement time).

The spatial and temporary nested model is implemented in C\#. It allows calculating temporal reliability indicators taking into account the influence of various factors and estimating the degree of their influence on the availability factor.

Keywords: radio-electronic nested systems, spatial and temporary model, terrain transport network, graph, attainability matrix, temporary route system.

\section{References}

1. Anisimov O.V., Belyakov R.A., Ignatev S.V., Kamensky I.E., Meshcheryakov V.D., Pristupyuk A.I., Tikhonov V.B., Chervakov V.O. Operation Basics of Radio Engineering Systems. Yaroslavl, 2015, 200 p.

2. Davydov P.S. Technical Diagnostics of Radio Electronic Systems. Moscow, Radio i svyaz Publ., 1998, 256 p.

3. Dorokhov A.N., Kernozhitsky V.A., Mironov A.N., Shestopalova O.L. Ensuring the Reliability of Complex Technical Systems. 2nd ed., St. Petersburg, Lan Publ., 2016, 352 p.

4. Anisimov O.V., Osipov A.A., Kharitonov A.V., Belyakov R.A. Maintenance of surface-to-air missile weapons in the aerospace defense system. Bulletin of Air Force Academy. 2012, iss. 15, part 1, pp. 34-38 (in Russ.).

5. Anisimov O.V., Popov T.A. The method of informational support of diagnosing complex technical complexes based on the patterns of elements of radioelectronic equipment electrical circuits. Proc. 2nd All-Russ. Sci. and Techn. Conf. "Theoretical and Applied Problems of Development and Improvement of Military Automated Control Systems". St. Petersburg, 2015, pp. 16-17 (in Russ.).

6. Anisimov O.V., Kurchidis V.A., Priveten A.S. A descriptive model of radio electronic equipment based on ontologies for automation of maintenance personnel information support in diagnosing complex technical complexes. High Tech in Earth Space Research. 2016, vol. 8, no. 3, pp. 72-77 (in Russ.).

7. Medvedev V.M., Mishchenko V.I., Solokha N.G. Development of the concept of product operation. Vestnik of OSU. 2008, no. 85, pp. 149-157 (in Russ.).

8. Cherkesov G.N. Evaluation of System Reliability with Regard to Spare Parts. St. Petersburg, BHV-Peterburg Publ., $2012,480 \mathrm{p}$.

9. Ignatev S.V., Tikhonov V.B., Krasnikov A.V., Osipov A.A. A spatio-temporal model of a material and technical resources traffic network of a radio-electronic facilities group exploitation. Software \& Systems. 2017, iss. 3, pp. $510-516$ (in Russ.).

10. Ore O. Theory of Graphs. American Mathematical Society Publ., 1962, 284 p. (Rus. ed.: Moscow, Nauka Publ., 1980, 336 p.).

11. Gorbatov V.A., Gorbatov A.V., Gorbatova M.V. Discrete Mathematics. Moscow, Astrel Publ., 2003, 447 p

12. Christofides N. Graph Theory: An Algorithmic Approach. Academic Press, 1975, 415 p. (Rus. ed.: Moscow, Mir Publ., 1978, 432 p.).

\section{Примеры бибциографического описания статьи}

1. Игнатьев С.В., Пцакса Ю.А., Красников А.В., Дрожжин А.В. Модель группировки радиоэлектронных систем дия оценки временных показателей надежности // Программные продукты и системы. 2018. T. 31. № 3. C. 598-604. DOI: 10.15827/0236-235X.123.598-604.

2. Ignatev S.V., Plaksa Yu.A., Krasnikov A.V., Drozhin A.V. A nested model of radio-electronic systems for estimation of temporary reliability. Software \& Systems. 2018, vol. 31, no. 3, pp. 598-604 (in Russ.). DOI: $10.15827 / 0236-235 X .123 .598-604$. 